\title{
High-Frequency Fourier Transform Ion Cyclotron Resonance Mass Spectrometry
}

\author{
Lutz Schweikhard*, George M. Alber, and Alan G. Marshall ${ }^{\dagger}$ \\ Department of Chemistry, The Ohio State University, Columbus, Ohio, USA
}

The experimental Fourier transform ion cyclotron resonance (FT/ICR) frequency range has been extended to $107 \mathrm{MHz}$. We report the observation of FT/ICR signals from electron-ionized species of mass-to-charge ratio $8,7,6,5,4,3,2$, and $1 \mu$ per elementary charge. We show that moderately high charge states of atomic ions $\left(\mathrm{e}, \mathrm{g} ., \mathrm{N}^{3+}\right)$ are easily generated and detected. Several applications for high-frequency FT/ICR mass spectrometry are proposed and discussed. (J Am Soc Mass Spectrom 1993, 4, 177-181)

\begin{abstract}
$\mathrm{E}$ xamples of the increased recent interest in ion cyclatron resonance (ICR) of ions of low massto-charge ratio, include precise mass measurement of elemental particles such as electrons (e) protons $(p)$, positrons $\left(e^{+}\right)$, and antiprotons $\left(p^{-}\right)$[1-3]. In addition, there is a need for broadband detection and monitoring of low mass-to-charge ratio ion clouds of mixed composition, such as those in (or delivered from) EBIS [4, 5] and EBIT [6, 7] instruments [8], or from accelerators $[9,10]$. Fourier transform ion cyclotron resonance (FT/ICR) mass spectrometry [11] provides, in principle, a universal method to achieve such broadband detection and monitoring. Detection of FT/ICR signals at high ICR orbital frequencies is also required for direct monitoring of ion-molecule reaction chemistry involving hydrogen ions. For example, the ion cyclotron orbital frequency of $\mathrm{H}^{+}$at a magnetic field of $7 \mathrm{~T}$ is above $100 \mathrm{MHz}$.

To date, the highest reported FT/ICR frequencies are $\sim 25 \mathrm{MHz}$ from ions of $\mathrm{m} / \mathrm{z} 3$ at $\sim 5 \mathrm{~T}$, in connection with determination of the mass difference between ${ }^{3} \mathrm{He}^{+}$and ${ }^{3} \mathrm{H}^{+}[12,13]$. However, commercial FT/ICR instruments are restricted to ICR frequencies below $10 \mathrm{MHz}$. In this paper, we report a significant extension of the ICR frequency range, with experimental detection of ICR signals up to more than $100 \mathrm{MHz}$. Fxperimental techniques and related aspects are discussed.
\end{abstract}

\footnotetext{
- Ohio State University Postdoctoral Fellow. Present address: Institute of Physics, Unuversity of Mainz, Staudinger Wèg 7, D-6500 Mainz, Germany.

${ }^{\dagger}$ Also a member of the Department of Biochemistry.

Address reprint requests to Alan G. Marshall, Department of Chemistry, The Ohio State University, 120 West 18 th Avenue, Columbus, $\mathrm{OH} 43210$.
}

\section{Experimental}

The FT/ICR mass spectrometer has been described briefly in a recent report [14] and will be described in detail in a forthcoming article [15]. The apparatus consists of a 7-T superconducting solenoidal magnet, an ion-pumped vacuum chamber, custom built ion trap, an electron gun located in the fringe field of the magnet, an Extrel (Madison, WI) 2000 data station, and associated custom-made switches, filters, mixers, and buffers.

Magnet. A 150-mm bore Oxford (Osney Mead, Oxford, England) superconducting magnet provides a magnetic field of $7 \mathrm{~T}$. The static magnetic field inhomogeneity, $\Delta B / B$, has been determined by a nuclear magnetic resonance probe to be less than $1 \mathrm{ppm}$ throughout a cylinder of $4-\mathrm{cm}$ length and $4-\mathrm{cm}$ diameter.

Vacuum chamber. An electropolished stainless steel vacuum chamber is pumped by a Perkin-Elmer (Norwalk, CT) TNB-X pumping station, comprising two sorption pumps, ten ion pumps ( $300 \mathrm{~L} \mathrm{~s}^{-1}$ pumping speed), and four titanium sublimation pumps that increase the pumping speed for removal of common residual gases. The entire vacuum chamber, including the interior ICR components (see below) was baked at $250^{\circ} \mathrm{C}$ for several days. The system achieves a base pressure of less than $6 \times 10^{-11}$ mbar (reading from a Bayert-Alpert gauge calibrated for nitrogen and enclosed by a custom-made magnetic shield), thereby providing for ultrahigh resolution FT/ICR/MS [14, 15]. The present experiments were performed at $\sim 2.5$ $\times 10^{-10}$ mbar by admitting the appropriate gases through an ultrahigh vacuum leak valve [Varian (Palo Alto, CA), model 9515106]. (Hydrogen gas was pro- 
duced by heating the sublimation pump filaments to moderate temperature.)

Ion trap and electron gun. The experiments reported below were performed with a homebuilt 2-in. cubic ion trap, constructed from OFHC copper plates (perforated to allow for better pumping) mounted on Macor insulators. The electron gun for electron impact ionization is mounted in the fringe field $(\sim 0.03 \mathrm{~T})$ of the magnet, and is displaced by $\sim 1.1 \mathrm{~m}$ from the trap along the magnetic field symmetry axis. Because the electron beam is tightly focused by electron cyclotron motion in the magnetic field, electron ionization in the trap produces an ensemble of ions whose initial ICR orbits are all centered very close to the magnetic field symmetry axis. The electron beam is collected behind the trap and monitored by a picoammeter, thereby ensuring that the trap is aligned with the symmetry axis of the magnetic field. The electron gun filament is made from rhenium (0.001 in. $\times 0.030 \mathrm{in}$. cross section). The electron beam was typically operated at an electron energy of $80 \mathrm{eV}$ (unless otherwise specified) at an electron current of $-1 \mu \mathrm{A}$ through the trap for several tens of milliseconds.

Experimental event sequence. An Extrel 2000 data station (Extrel FTMS, Madison, WI) controls the experimental event sequence as well as data presentation and storage. The standard event sequence (consisting of quench, ionization, delay, excitation, and detection events) has been modified to allow for a more extended electron beam ionization period (necessitated by the very low neutral pressure). The frequency range $(0-6 \mathrm{MHz})$ of the commercial instrument has been extended by incorporation of an external frequency synthesizer (PTS-160, Programmed Test Sources, Littleton, MA) and a power amplifier (model 25A100, Amplifier Research, Souderton, PA). The excitation signal is turned on and off by a custom-made switch. Two excitation signals of opposite phase (for the two opposed excitation electrodes) are produced by passing the signal through a transformer with a grounded center tap. The ion signal is differentially detected by the two remaining side plates, amplified by a homebuilt preamplifier [15], and heterodyned down to a much lower frequency (several hundred hertz) by passing through a mixer (model 10534A, Hewlett-Packard, Palo Alto, CA) and low-pass filter, and then fed to the analog-to-digital converter input of the data station. The excitation signal serves a second purpose as a reference for the heterodyne detection. For this reason, ions are excited a little off resonance but well within the Fourier bandwidth of the singlefrequency if excitation pulse. Another advantage of this approach is that signal averaging (i.e., addition of time-domain signals from successive event sequences) can be performed without phase synchronization of the excitation waveforms from successive event se- quences. Any phase shift of the excitation signal results in a corresponding phase shift of the detected IC $\mathrm{K}$ signal (so long as the excitation and delay periods remain fixed from one acquisition to the next); thus, the net phase difference between them does not change, and the heterodyned signal phase is constant, independent of the phase of the excitation signal. In the present experiments, the ion signal was strong enough to work with a single transient, and it was not necessary to take advantage of the above signal-averaging capability.

Heterodyne techniques in ICR are not new, having been used in the first FT/ICR experiment [16] and in various subsequent high-frequency single-ion Penning trap measurements [17]. However, the present method is adapted to widely available commercial FT/ICR/MS instruments, and moreover lends itself to the use of multiple-heterodyne excitation and detection, so as to be able to excite and detect ions of widely separated mass-to-charge ratio simultaneously at high digital resolution [14], as will be explained in full detail in a future paper.

\section{Results and Discussion}

\section{High-Frequency FT/ICR Spectra}

Figures 1 and 2 show FT/ICR magnitude-mode mass spectra of several low mass ions. We have observed singly charged $\mathrm{H}^{+}, \mathrm{H}_{2}{ }^{+}$(not shown), and $\mathrm{H}_{3}{ }^{+}$(from hydrogen gas released from the sublimation pump); $\mathrm{D}^{+}, \mathrm{D}_{2}^{+}, \mathrm{D}_{3}^{+}$(from deuterium gas admitted through a leak valve), $\mathrm{DH}^{+}, \mathrm{DH}_{2}{ }^{+}$(not shown), and $\mathrm{D}_{2} \mathrm{H}^{+}$ (from deuterium and hydrogen gases leaked in); as well as doubly charged nitrogen and oxygen atomic ions, $\mathrm{N}^{2+}$ and $\mathrm{O}^{2+}$. Typical mass resolving power, $m / \Delta m$ (in which $\Delta m$ is defined as the magnitude-mode full line width at half peak height) is $\sim 10^{7}$, and appears to be limited mainly by pressure and space charge. Note that each reported ICR orbital frequency was measured under slightly different conditions (e.g.,

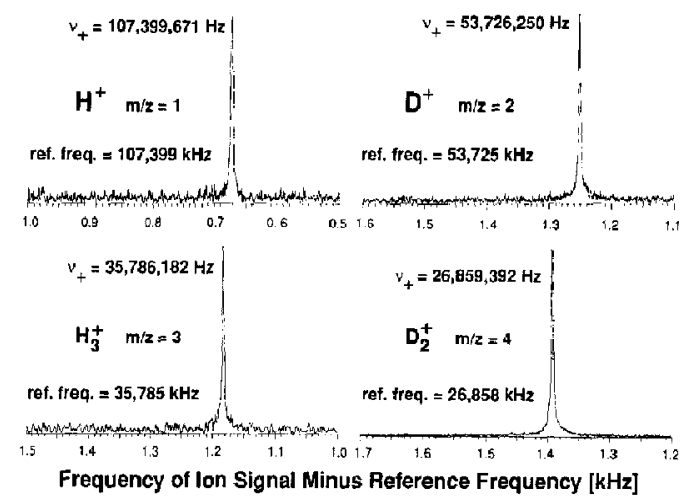

Figure 1. Magnitude-mode FT/ICR mass spectra of $\mathrm{H}^{+}, \mathrm{D}^{+}$, $\mathrm{H}_{3}^{+}, \mathrm{D}_{2}^{+}$at $7.0 \mathrm{~T}$. 


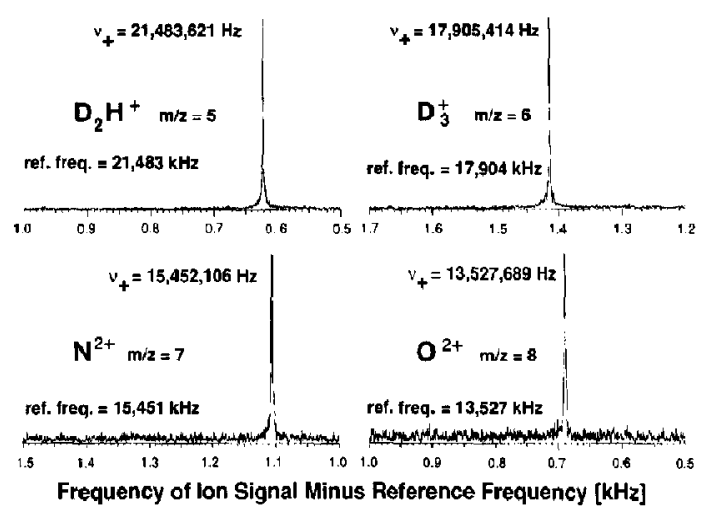

Figure 2. Magnitude-mode FT/ICR mass spectra of $\mathrm{D}_{2} \mathrm{H}^{+}$, $\mathrm{D}_{3}^{+}, \mathrm{N}^{2+}$, and $\mathrm{O}^{2+}$ at $7.0 \mathrm{~T}$.

ICR orbital radius, number of ions); thus, the ratio of the ICR orbital frequencies (corrected for the shift from the electric trapping field) for any two of the spectra does not match exactly to the ratio of the corresponding ion masses.

\section{Formation of $\mathrm{D}_{3}{ }^{+}$by Electron Bombardment of Deuterium Gas}

An appliçation of high-frequency monitoring for the study of ion-molecule reactions is demonstrated by observing the relative abundance of trapped $\mathrm{D}_{3}{ }^{+}$ions as a function of trapping period. Figure 3 shows the time evolution of the ICR signals from $\mathrm{D}^{+}, \mathrm{D}_{2}{ }^{+}$, and $\mathrm{D}_{3}{ }^{+}$produced by a short electron beam pulse $(20 \mathrm{~ms}$, electron current through trap of $\sim 1.7 \mu \mathrm{A}$ ), after which the trapped ions react with neutral background gas for a period of up to $44 \mathrm{~s}$. The pressure was about $2 \times$ $10^{-10}$ inbar (recalibrated for hydrogern) corresponding to a collision rate of the order of $10^{-4} \mathrm{~s}^{-1}$ for neutral thermal particles. The data shown in Figure 3 were taken at a trapping potential of $8 \mathrm{~V}$; therefore, at least some trapped ions may have higher than thermal energy. Lowering the trapping potential down to $2 \mathrm{~V}$ did not change the exponential decay time constant for the $\mathrm{D}_{2}{ }^{+}$signal. However, ions may also gain energy from the ionization process itself. At any rate, the present observations show that the cross section for collisions of the ions with the neutral background gas appears to be several orders of magnitude higher than that of thermal neutral-neutral collisions. Moreover, Figure 3 confirms that $\mathrm{D}_{3}{ }^{+}$is formed from $\mathrm{D}_{2}{ }^{+}$rather than $\mathrm{D}^{+}$, because the rate of appearance of $\mathrm{D}_{3}{ }^{+}$parallels the rate of disappearance of $\mathrm{D}_{2}{ }^{+}$, whereas $\mathrm{D}^{+}$remains at the same relative abundance throughout the reaction period.

\section{Formation of $\mathrm{N}^{3+}$ by Electron Impact Ionization}

Finally, it is worth noting that moderately charged atomic ions may easily be produced by standard operation of the FT/ICR instrument. Figure 4 shows an FT/ICR mass spectrum of $\mathrm{N}^{3+}$, produced by a 4-s,
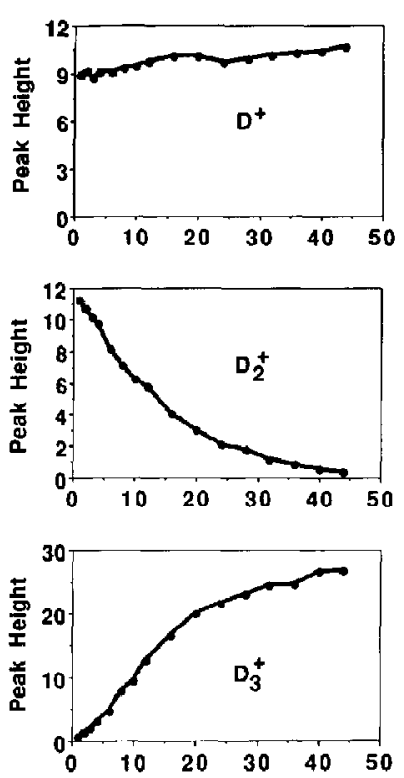

Ion-Molecule Reaction Period (s)

Figure 3. Relative abundances of $\mathrm{D}^{+}, \mathrm{D}_{2}{ }^{+}$, and $\mathrm{D}_{3}{ }^{+}$as a function of ion-molecule reaction period between electron impact ionization and excitation/detection events, showing the buildup of $\mathrm{D}_{3}{ }^{+}$by reaction of $\mathrm{D}_{2}{ }^{+}$with neutral $\mathrm{D}_{2}$ background gas. The data points have been connected by straight lines to guide the eye. Because each data set was acquired independently, the ordinate values are not directly comparable.

6- $\mu \mathrm{A}$ electron beam ( $80-\mathrm{eV}$ electron energy). The FT/ICR magnitude-mode spectrum has a resolving power of $\sim 100$ million. (For this measurement the pressure was lowered to $\sim 1 \times 10^{10}$ mbar.) Figure 5 shows the signal magnitude as a function of electron energy (reported as the offset potential of the heated filament that produces the electrons). The values in Figure 5 are the filament potential values requested from the data station. Note: If $50 \mathrm{~V}$ is requested from the data station, potentials of $\sim 51$ and $\sim 53 \mathrm{~V}$ are measured on either end of the filament, indicating that the potential drop along the filament is $\sim 2 \mathrm{~V}$. Furthermore, the elcctrons gain a little more kinetic energy during their flight through the trap: a maximum

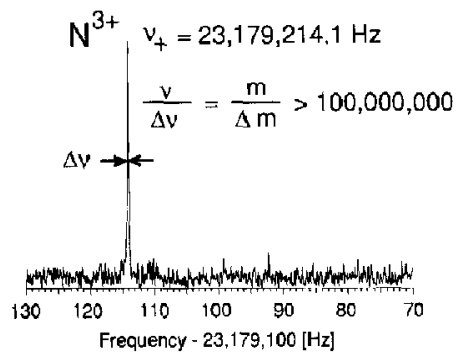

Figure 4. Ultrahigh-resolution magnitude-mode FT/ICR mass spectrum of $\mathrm{N}^{3+}$. 


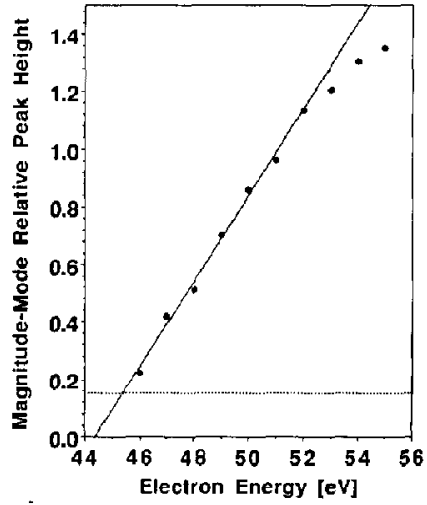

Figure 5. $\mathrm{N}^{3+}$ FT/ICR magnitude-mode mass spectral relative peak height as a function of the electron energy requested from the data station. The dashed line indicates the noise level. The straight line results from a linear fit to the data points from 46 to $51 \mathrm{~V}$.

of $\sim 4 \mathrm{eV}$ at either trapping electrode and $\sim 1.3 \mathrm{eV}$ at the trap center (for a trapping plate voltage of $4 \mathrm{~V}$ and grounded side plates; see, for example, ref 18). Corrected for these two effects, the "appearance potential" of $\mathrm{N}^{3+}$ is $\sim 49 \mathrm{~V}$, in agreement with detailed cross section studies [19] and the known third ionization energy of nitrogen of $47.5 \mathrm{eV}[20]$, indicating that these ions are formed by further electron impact ionization of previously formed $\mathrm{N}^{2}$ ions. Similar studies (not shown) of the appearance potential for $\mathrm{N}^{2+}$ show that these ions are formed by means of electron impact ionization of $\mathrm{N}^{+}$. Note that such experiments offer a simple means for distinguishing a true ICR signal from harmonics arising from ions of higher mass-to-charge ratio (e.g., $\mathrm{N}^{2+}$ versus second harmonic of $\mathrm{N}^{+}$). Alternatively, harmonics may also be identified from the frequency shift as a function of trap voltage [21]. Charge state determination by the isotopic distribution [22] is possible for atomic ions only if there is more than one isotope for the element under investigation.

\section{Future Experiments Based on High- Frequency FT / ICR / MS}

Ionization methods based on high-energy light ions. The experiments described above open a new field for FT/ICR investigations. As pointed out recently [23], light ions can be excited to very high kinetic energy and still be confined within the trap. For example, $\mathrm{H}^{+}$ ions at an ion cyclotron orbital radius of $1 \mathrm{~cm}$ in a $7-T$ magnetic field have a kinetic energy in excess of 200 $\mathrm{keV}$ ! Collision of a high-energy light ion with a heavy target will deposit most of that energy in the centerof-mass reference frame. Excited light ions passing through clouds of heavy neutrals or ions might then be expected to produce significant ionization and fragmentation and to deposit considerable energy that could lead to further dissociation, thermionic emission, and so forth. Alternatively, the excited light ions could be directed onto the trap electrodes as in recent FT/ICR experiments on surface-induced dissociation [24, 25 ] - not with the intent to break the colliding ion into pieces, but rather to deposit its energy on the surface and cause a desorption of secondary ions as in secondary ion mass spectrometry. Combination of these features with the $\mathrm{MS}^{n}$ capabilities of FT/ICR [11] is expected to lead to exciting new experiments.

ICR orbital radius from relativistic frequency shift. Another aspect of high-frequency FT/ICR is that the high velocity of the excited ions leads to a relativistic mass shift. The resulting ICR orbital frequency shift can be used for accurate determination of the ion cyclotron orbital radius [26]. For example, the post-excitation ion cyclotron orbital radius is exactly predictable from the (resonant) excitation voltage amplitude and duration, for ions in the midplane $(z=0)$ of an orthorhombic or cylindrical ion trap [18, 27]. A simple test for such a model is to compare the predicted and experimentally measured maximum ICR orbital radius at which ions are ejected from the trap. Although the onset of radial ejection matches the theory precisely, it is found experimentally that a larger excitation voltage-amplitude producl is required for complete ejection [28, 29]. The relativistic shift may offer the needed independent means for determining ICR orbital radius required to test explanations of that phenomenon. Moreuver, the (large) relativistic shift for low-mass ions may be used to calibrate such ICR orbital radius measurements for higher mass ions of chemical and biological interest, because the spatial dependence of the electric field is the same at all frequencies, and the ICR signal strength is directly predictable from the amplitude and time dependence of the rf electric excitation field $[18,27$, 30].

FT/ICR mass spectral peak shape. Recent theoretical calculations [31] show that ions initially formed at different positions along the trap $z$ axis should exhibit wide (up to 20\%) variation in postexcitation cyclotron orbital radius, and thus should exhibit substantial inhomogeneous line broadening due to a distribution in relativistic frequency shift for low-mass ions. However, the experimentally observed FT/ICR mass spectral peak shape in a cubic trap under these conditions can range from a quite narrow $(<1 \mathrm{~Hz}$ ) single peak to broad or split peaks. Experimental tests of explanations of these effects depend on means for measuring trapping oscillation amplitude [32] and ion cyclotron orbital radius. However, prior ICR orbital radius determination methods are valid only for ions confined to the $z=0$ trap midplane [28, 29,33]. Because low-mass ions (rendered observable by the techniques described in this paper) exhibit relativistic cyclotron frequency shifts up to $1,000 \mathrm{~Hz}$ when excited to an orbital radius of $\sim 1 \mathrm{~cm}$, such shifts offer a direct measure of postex- 
citation ion cyclotron radial dispersion arising from polydisperse ion initial $z$ distribution and/or nonquadiupolar trapping potential in ICR ion traps of conventional or new design.

Coulumb shift and broadening of FT $/ I C R$ mass spectral peaks. Theoretical models for the shifting and broadening of FT/ICR mass spectral peaks are in a rapid state of development [34-37]. Those models are based on presumed prior knowledge of the radial and axial distribution of a large number of ions of the same or different mass-to-charge ratio. Here, again, the relativistic frequency shift and peak broadening for lowmass ions should offer a sensitive measure of the radial distribution of ions, thereby providing a means for distinguishing between various theoretical models for Coulomb shifting and broadening.

Multiple ion detection. Finally, the present measurements have all been performed in heterodyne mode for high-resolution detection of ions of one mass-to-charge ratio at a time. Simultaneous monitoring of ions of two or more mass-to-charge ratios can be achieved by undersampling [38] and /or multiple heterodyning methods [15, 39]. Alternatively, with fast analog-to-digital converters [e.g., 200 megasamples/sec at 8 bit (Model AD770, Analog Devices, Norwood, MA)], broadband detection should be possible.

\section{Acknowledgments}

We thank T. Hickey for his help in construction of the 2 -in. ICR ion trap and T. L. Ricca for design, construction, and testing of high-frequency electronics. This work was supported by grants (to A. G. M.) from the National Science Foundation (CHE-9021058) and The Ohio State University. L. S. thanks The Ohio State University and the Deutsche Forschungsgemeinschaft for postdoctoral fellowships.

\section{References}

1. Van Dyck, R. S. J.; Moore, F. L.; Farnham, D. L.; Schwinberg, P. B. Int. J. Mass Spectrom. Ion Proc. 1985, 66, 327-337.

2. Schwinberg, P. B;; van Dyck, R. S. Jr.; Dehmelt, H. G. Phys. Lett. A 1981, 81, 119-120.

3. Gabrielse, G.; Fei, X.; Orozco, L. A.; Tjoelker, R. L.; Haas, J.; Kalinowsky, H.; Trainor, T. A.; Kells, W. Phys. Rev. Lett. 1989, 63, 1360-1363.

4. Donets, E. D. Physica Scripta 1983, T3, 11-18.

5. Arianer, J.; Cabrespine. A.; Goldstein, C. Nucl. Instr. Meth. 1982, 193, 401-413.

6. Levine, M. A.; Marrs, R. E.; Henderson, J. R.; Knapp, D. A.; Schneider, M. B. Physica Scripta 1988, T22, 157-163.

7. Beiersdorfer, P. Nucl. Instrtum. Meth. 1991, B56/57, 1144-1147.

8. Schmieder, R. W. In Physics of Highly lonized Atoms; NATO Advanced Studies Institutes, Ser. B; R. Marrus, Ed; Plenum: New York, 1990; Vol. 201, pp. 321-376.

9. Beyer, H. F.; Bollen, G.; Bosch, F.; Egelhof, P.; Franzke, B.; Hasse, R. W.; Kluge, H.-J.; Kozhuharov, C.; Kühl, T.; Liesen, D; Mann, R.; Mokl, P. H.; Müller, A.; Müller, R. W.; Münzenberg, G.; Poth, H.; Schweikhard, L.; Schuch, R.; Werth, G. "HITRAP, A Facility for Experimentation with
Trapped Highly-Charged Ions at Gesellschaft für Schwerionen forschung Darmstadt, Germany," GSI, 1990.

10. Poth, H.; Hasse, R. W. In 19th Int. Symp. on "Cooler Rings and Their Applications"; Tokyo, 1990; T. Katayama and A. Noda, Eds. World Scientific Singapore, 1991; pp. 108-123.

11. Marshall, A. G; Schweikhard L. Int. J. Mass Spectrom. Ion Proc. 1992, 118/119, 37-70.

12. Lippmaa, E. T.; Pikver, R.; Suurmaa, E.; Past, J.; Puskar, J.; Koppel, I.; Tammik, A. Phys. Rev. Lett. 1985, 54, 285-288.

13. Talrose, V. L.; Nikolaev, E. N. Ado. Mass Spectrom. 1985, 10A, 343-357.

14. Hill, N. C.; Alber, G. M.; Schweikhard, L.; Ricca, T. L.; Marshall A. G. In Proc. 39th Am. Soc. Mass Spectrom. Conf, on Mass Spectrometry \& Allied Topics; Am. Soc. Mass Spectrom.: Nashville, TN, 1991; pp. 1501-1502.

15. Alber, G. M.; Marshall, A. G.; Hill, N. C.; Schweikhard, L; Ricca, I'. L.; unpublished results.

16. Comisarow, M. B.; Marshall, A. G. Chem. Phys. Lett. 1974, 25. 282-283.

17. Van Dyck, R. S. Jr.; Schwinberg, P. B. Phys. Rev. Lett. 1981, $47,395-398$.

18. Grosshans, P. B.; Marshall, A. G. Anal Chem. 1991, 63 , 2057-2061.

19. Tawara, H.; Kato, T. Atom. Data Nucl. Data Tables 1987, 36. $167-353$.

20. Handbook of Chemistry and Physics; 71st ed.; Lide, D. R., Ed.; CRC Press: Boca Raton, FL, 1990; pp. 10-210.

21. Limbach, P. A.; Schweikhard, L.; Cowen, K. A.; McDermott, M. T.; Marshall, A. G.; Coe, J. V. J. Am. Chem. Soc. 1991, 113, 6795-6798

22. Henry, K. D.; Quinn, J. P.; McLafferty, F. W. J. Am. Chem. Soc. 1991, 113, 5447-5449.

23. Schweikhard, L.; Alber, G. M.; Marshall, A. G. Physica Scripta 1992, 45, in press.

24. Ijames, C. F; Wilkins, C. L. Anal. Chem. 1990, 62, 1295-1299.

25. Williams, E. R; Henry, K. D.; McLafferty, F. W.; Shabanowitz, J.; Hunt, D. F. I. Am. Soc. Mass Spectrom. 1990, 1, 413-416.

26. Schweikhard, L.; Alber, G. M.; Marshall, A. G., unpublished resilts.

27. Grosshans, P. B.; Marshall, A. G. Int. J. Mass Spectrom. Ion Proc. 1990, 100, 347-379.

28. Grosshans, P. B.; Shields, P. J.; Marshall, A. G. J. Chem. Phys. 1991, 94, 5341-5352.

29. Grosshans, P. B.; Marshall, A. G. Int. J. Mass Spectrom. Ion Proc. 1992, 115, 1-19.

30. Guan, S. J. Am. Soc. Mass Spectrom. 1991, 2, 483-486.

31. Chen, R.; Grosshans, P. B.; Marshall, A. G., unpublished results, 1992

32. Dunbar, R. C; Weddle, G. H. I. Phys. Chem. 1988, 92, 5706-5709.

33. Grosshans, P. B.; Shields, P. J.; Marshall, A. G. J. Am. Chem. Soc. 1990, 112, 1275-1277.

34. Wang, T.-C. L.; Marshall, A. G. Int. J. Mass Spectrom. Ion Proc. 1986, 68, 287-301.

35. Chen, S.-P.; Comisarow, M. B. Rapid Commun. Mass Spectrom. 1991, 5, 450-455.

36. Chen, S.-P.; Comisarow, M. B. Rapid Commun. Mass Spectrom. 1992, 6, 1-3.

37. Beu, S. C.; Hendrickson, C. L.; Laude, D. A. Jr. In Proc, 40 th Am. Soc. Mass Spectrom. Ann. Conf. on Mass Spectrom. \& Allied Topics; Am. Soc. Mass Spectrom.: Washington, DC, 1992; pp. TP75.

38. Wang, M.; Marshall, A. G. Anal. Chem. 1988, 60, 341-344.

39. Rempel, D. L.; Ledford, E. B. Jr.; Sack, T. M.; Gross, M. L. Anal. Chem. 1989, 749-754. 